\title{
TITLE: PROGRESS WITH THE SLOTTED-TUBE PULSED MICROWIGGLER
}

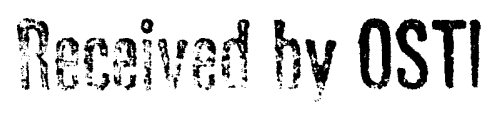

OCT 041991

\section{AUTHOR(S): Roger W. Warren}

\section{SUBMITTED TO: Thirteenth International Free-Electron Laser Conference Santa Fe, NM August 25-30, 1991}

\section{DISCLAIMER}

\begin{abstract}
This report was prepared as an account of work sponsored by an agency of the United States Government. Neither the Ut.ited States Government nor any agency thereof, nor any of their employees, makes any warranty, express or implied, or assumes any legal liability or responsibility for the accuracy, completeness, or usefulness of any information, apparatus, product, or process disclosed, or represents that its use would not infringe privately owned rights. Reference herein to any specific commercial product, process, or service by trade name, trademark, manufacturer, or otherwise does not necessarily constitute or imply its endorsement, recommendation, or favoring by the United States Government or any agency thereof. The views and opinions of authors expressed herein do not necessarily state or reflect those of the United States Government or any agency thereof.
\end{abstract}

By acceptance of this article, the publisher recognizes that the U.S. Government retains a nonexclusive, royalty-free license to publish or reproduce the published form of this contribution, or to allow others to do so, for U.S. Government purposes.

The Los Alamos National Laboratory requests that the publisher identify this article as work performed under the auspices of the U.S. Department of Energy.

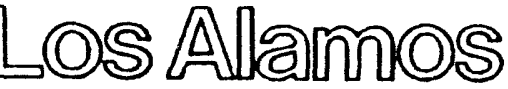

Los Alamos National Laboratory

Los Alamos, New Mexico 87545 


\title{
PROGRESS WITH THE SLOTTED-TUBE PULSED MICROWIGGLER* \\ Roger Warren
}

Los Alamos National Laboratory

Los Alamos, New Mexico 87545

\begin{abstract}
A pulsed microwiggler can produce a wiggler field of unusually short period and high strength. A period of a few millimeters and a field of 5 appear possible. A satisfactory design is hard to realize, however, for many reasons: the high current, the current nonuniformities caused by the skin effect, thermal stresses in the parts, the high precision, etc. In addition, measuring the field with adequate resolution in the time domain, as well as in all three spatial coordinates, is challenging, particularly inside the small bore of a microwiggler. This paper presents recent design modifications that illustrate new solutions to these problems, and details of the construction, testing, and performance of wigglers already built.
\end{abstract}

\section{INTRODUCTION}

The gain of a free-electron laser depends strongly on the K-value of its wiggler, where $\mathrm{K}=0.93^{*}$ period $(\mathrm{cm}) *$ field $(\mathrm{T})$. A $\mathrm{K}$-value near 1 is ideal for most uses, but higher $\mathrm{K}$-values are needed to generate harmonics efficiently. The field of permanent magnets is limited to about $1 \mathrm{~T}$; therefore, a wiggler period of about $1 \mathrm{~cm}$ is the shortest that can be usefully built with permanent magnets. For practical reasons, a limiting period of about $3 \mathrm{~cm}$ is more common.

*Work supported by Los Alamos National Laboratory Insitutional Supporting Research, under the auspices of the United States Department of Energy and the Office of Basic Energy Sciences, Division of Advanced Energy Projects. 
A useful alternative to a permanent-magnet wiggler is a pulsed electromagnetic one. Very high fields can be obtained for short times with various configurations of conductors. Ultimately, either heating of the conductors or the large stresses on them limit the field. Periods as short as 1 to $2 \mathrm{~mm}$ with a $\mathrm{K}$-value of 1 appear possible. A previous paper [1], reports our development of a pulsed wiggler based on a wound double-helix conductor configuration. A more recent paper [2] reports calculations and initial construction of a very different wiggler with a slottedtube conductor configuration. The slotted-tube conductor provides several advantages over the wound helix: it generates plane-polarized rather than circularly polarized light; it does not need a support inside the winding; correct fields at the ends are easy to achieve; cooling is better; its inductance is less; and the electrical connections are simpler to make.

This paper relatesthe author's further experience with this slotted-tube design, in particular, the engineering problems encountered, solutions to them, the present status, and a view of the future of this wiggler type

\section{DESIGN CONCEPT}

Figure 1 simplifies the construction of the slotted-tube wiggler system. There are only four parts: an inner cylinder (the wiggler), an outer cylinder (the current return path), a plug connecting the cylinders at one end, and connections from the cylinders to a power supply at the other end. No field would be generated inside the wiggler by the currents in these symmetric coaxial conductors except for the periodic slots cut on top and bottom of the wiggler. The slots divert the current and generate strong transverse fields (horizontal in Fig. 1) of the kind needed in a wiggler.

Various slot shapes have been investigated. Figure 2 shows four kinds cut in an $\sim 3 \mathrm{~mm}$ (1/8 in.)-o.d. copper tube by spark machining techniques: 2 -mil-wide straight slots; 12-mil-wide straight slots; V-slots; and curved-T slots. Also shown are 2-mil- 
wide slots cut in an $1.5 \mathrm{~mm}$ tube. Each slot configuration has advantages. The simple slots are easy to make. The wide ones allow the field to penetrate quickly from the outside of the wiggler tube to its inside. The narrow slots generate smaller wakefields as electrons pass. The $T$ slots reduce the current density and, therefore, excess heating at the tip of the slots. These effects, that is, field penetration, wake-fields, heating, and field strength, dominate design considerations.

\section{ENGINEERING DESIGN}

The distribution of current in the slotted tube and the field that it produces is difficult to calculate, particularly if three-dimensional, time-dependent factors are included. The static problem has been solved analytically for a straight, thin slot in a thin tube [3] and on a computer for more complex slots in a thin tube. Measurements of the fields produced by these arrangements in enlarged models that use aluminum foil for the conductor and Hall-field probes agreed with the. calculation. Measurements of the field produced in real pulsed microwigglers also agreed with the calculation.

Figure 3 shows the value of $\mathrm{K}$ produced by a thin tube wiggler with different values of $S$, the ratio of circumference to period, with simple slots of ideal depth and with a current of $25 \mathrm{kA}$ flowing through it. More complex slots produce fields that can exceed those of fig. 3 , but only by about $25 \%$. The ideal depth is discussed below. For several reasons, we usually choose a value for $S$ near 1 . The value of $K$ is then near unity. Thus, for a wiggler of any period, the current needed for $K=1$ and $R=1$ is about $25 \mathrm{kA}$. If the diameter of the wiggler tube were increased without changing the period, for example, to provide room for another tube within it, the value of $R$ would increase and the current needed to produce $K=1$ would increase rapidly. 
This extra tube appears to be essential to provide a vacuum enclosure for the electron beam and to exclude the water used for cooling. However, the large reduction in field and complexity in design that the extra tube introduces, prompted the decision to eliminate it and to solve the vacuum and cooling problems in another way. Various glasses, enamels, and epoxy glues bond well to copper and can be used to fill the slots. Then the copper conductor will act as its own vacuum enclosure.

\section{SUPPLYING THE CURRENT}

The resistance and inductance of a typical wiggler system are $1 \mathrm{~m} \Omega$ and $50 \mathrm{nH}$. These impedance values present two problems: the internal impedance of the pulsedpower supply, and the impedance of the connecting cables exceed greatly $1 \mathrm{~m} \Omega$, makes operation inefficient. In addition, the time constant, $L / R$, which determines the rise time of the current in the wiggler is too long, $\sim 50 \mu \mathrm{s}$, for many. Connecting a transformer between the wiggler and the power supply correct the impedance problem. Figure 4 illustrates the compact arrangement [4] used with the transformer to reduce the system's inductance. The size of the transformer core is chosen to generate a 100-ps pulse. The single-turn secondary is composed of the wiggler, the return tube, and the close-fitting sheet-metal cover surrounding the core. The transformer has two primary windings of ten turns each. One of the primary windings is connected to a pulse-forming network (PFN) of $0.1 \Omega$ internal impedance delivering the 2 to $3 \mathrm{kA}$ current pulse. The other is connected through an inductor to a 2-A current supply to prebias the uncut core and thus allow the maximum pulse length before core saturation occurs.

Lowering the circuit's inductance can reduce the $L / R$ time constant of the wiggler system. This alteration is most easily made by shrinking the outer returnpath cylinder around the inner wiggler tube. The plan is to cool botk cylinders by flowing water in the gap between them, so there is a conflict between inductance- 
reduction and water flow-restriction. A gap of $0.5 \mathrm{~mm}$ will give an inductance of $\sim 10$ nH. Increasing $R$ can further reduce the time constant An external series-connected resistance will accomplish this goal, but at the expense of increased demand on the power supply.

\section{OTHER CRITICAL TIME CONSTANTS}

If one were able to supply a perfectly square current pulse to the wiggler, its field would not be constant during the pulse. There are at least three different reasons for this result: For very early times in the pulse, the field is confined to the space between the wiggler and the return tube. The slots act as tiny capacitors, passing unattenuated the high-frequency components of the pulse. To develop a field on the axis of the wiggler, the field must penetrate from the outside of the wiggler tube to the inside through the slots. This penetration takes 1 to $10 \mu \mathrm{s}$, depending on the width, shape, and orientation of the slots.

When the field is established inside the wiggler, current flows on both the inside and outside surfaces of the wiggler tube and on the sides of the slots. It takes from 5 to $20 \mu \mathrm{s}$, depending on the thickness of the tube and the material of its construction, for the current distribution in the wiggler to smooth out and fill the conductor's volume. During this time, the field intensity and shape will shift moderately.

Toward the end of the pulse, conductor heating becomes important. It shows up first where the current density is the greatest, at the tip of the slot. The temperature can be lowered considerably by using a "softer" slot like the curved $\mathrm{T}$ slot. The major consequence of this kind of localized heating is an increase in resistance at the tip of the slot that shifts the current away from the siot. The shift introduces a quadrupole moment to the field that exerts a focusing or defocusing force on the electron beam. The length of the slot was initially carefully chosen and carefully cut to have the 
ideal length for which no quadrupole field is generated. Upsetting this delicate balance can have serious effects on the electron beam.

\section{MANUFACTURING TOLERANCES}

The major tolerance issues are control of the wall thickness of the wiggler tube and control over the shape and position of the slots. Many of our wigglers are made from common 3-mm (1/8-in) tubing. Because it is made in large quantities, we can easily select samples with relatively uniform walls. Without selection, the thickness can vary by $10 \%$ from one side of the wiggler to the other. Figure 5 shows a measurement of the (integral of the) field on the axis of a wiggler made with such a tube. Its slope is caused by wall nonuniformity. The nonuniform wall has caused the current to flow more strongly on one side of the tube than on the other and to generate a uniform transverse field that would deflect electrons with an ever-increasing angle.

The slots are cut with a spark-erosion technique on a numerically controlled machine. If the wiggler tube is perfectly straight, the slots will be positioned perfectly with a constant depth. If, however, the tube is bent, their depth will vary with position. This variation creates transverse fields that bend the electron beam seriously. Currently special tubes are being obtained that are very straight and that have better wall uniformity.

\section{PERFORMANCE OF TESTED MODELS}

Testing the performance of microwigglers is not a trivial pursuit. The field probe must be small enough to fit into the bore of the wiggler and to have adequate time resolution to provide information about the relative field strengths during the various time-dependent events previously described. The pulsed-wire technique, described in these proceedings and elsewhere $[5,6]$, was used. We were easily able to monitor the L/R time constant, the time constant governing the penetration of the fields through the slots, and the heating time constant. 
Aberrations in the field caused by imperfectly made wigglers were measured and then corrected by drilling small holes in the return tube to modify its current distribution. We also measured the quadrupole fields in such a wiggler by displacing the wire used in the measuring technique various distances from the wiggler's axis. Using the transformer to multiply the current available from the PFN, we have reached a current of $20 \mathrm{kA}$ with the short-period (3- $\mathrm{mm}$ ) simple-slit wiggler system. This current produced a peak field of $2 T$, a peak $K$ value of 0.5 , and an rms $K$ value of 0.4. A higher current and field were not reached because of the combined effects of a poorly chosen $\mathrm{S}$ value, insufficient core area in the transformer, and excessive inductance of the wiggler. Modifications that should easily allow attainment of an rms $K$ value of 1 are being made to all three. The average temperature rise in the wiggler for this current was less than $10 \mathrm{~K}$ for a 100 - $\mu$ s-long pulse. We have not yet devised a good way to measure the local temperature rise near a slot and have not installed cooling provisions on this wiggler. We have, therefore, not tried to exceed this field or to achieve a high repetition rate.

\section{CONCLUSIONS}

We have investigated the physics and engineering issues posed by microwiggler systems, chosen an acceptable geometry for the current conductors, solved most of the engineering problems encountered, built several prototype systems, and have measured the fields produced. We have achieved a $\mathrm{K}$ value of $\sim 0.5$ with a wiggler period as short as $3 \mathrm{~mm}$ for a $100 \mu$ s pulse. This combination of short period and high $K$ value is about a factor of 10 better than that achieved with permanent magnet wigglers. We intend to achieve $K$ values greater than 1 with this wiggler, to extend this level of operation to high repetition rates, and to achieve even higher $K$ values and shorter periods by reducing the pulse length to 10 to $20 \mu \mathrm{s}$. 


\section{REFERENCES}

1. "High-Field Pulsed Microwigglers," R. W. Warren, D. W. Feldman, and D. Preston, Nucl. Instr. and Meth. A296 (1990), 558.

2. "Design Considerations for Pulsed Microwigglers," R. W. Warren, Nucl. Instr. and Meth. A304 (1991), 765.

3. R. L. Gluckstern, "Slotted Cylindrical Shell for Use as a Compact Wiggler," submitted to Phys. Rev.

4. Transformer built by Stangenes Industries, Inc., 1052 East Meadow Circle, Palo Alto, CA 94303.

5. R. W. Warren and C. J. Elliott, "A New System for Wiggler Fabrication and Testing, in Undulator Magnets for Synchrotron Radiation and Free Electron Lasers, Eds. R. Bonifacio, L. Fonda, and C. Pellegrini, (World Science Publication Co. 1988, 28.

6. R. W. Warren, "Limitations on the Use of the Pulsed-Wire Field Measuring Technique," Ilucl. Instr. and Meth. A272 (1988), 257.

\section{FIGURE CAPTIONS}

1. Simplified diagram of a pulsed-wiggler system.

2. Various slotted tubes used.

3. K vs $\mathrm{S}$ for a simple slot.

4. Diagram illustrating transformer use.

5. Field measurement of a wiggler with nonuniform walls. 
$\frac{8}{8}$
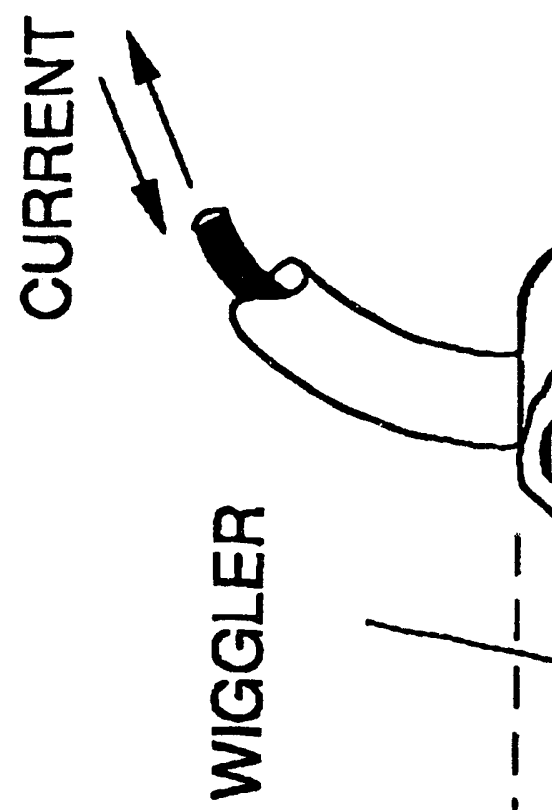


$$
\triangleq
$$




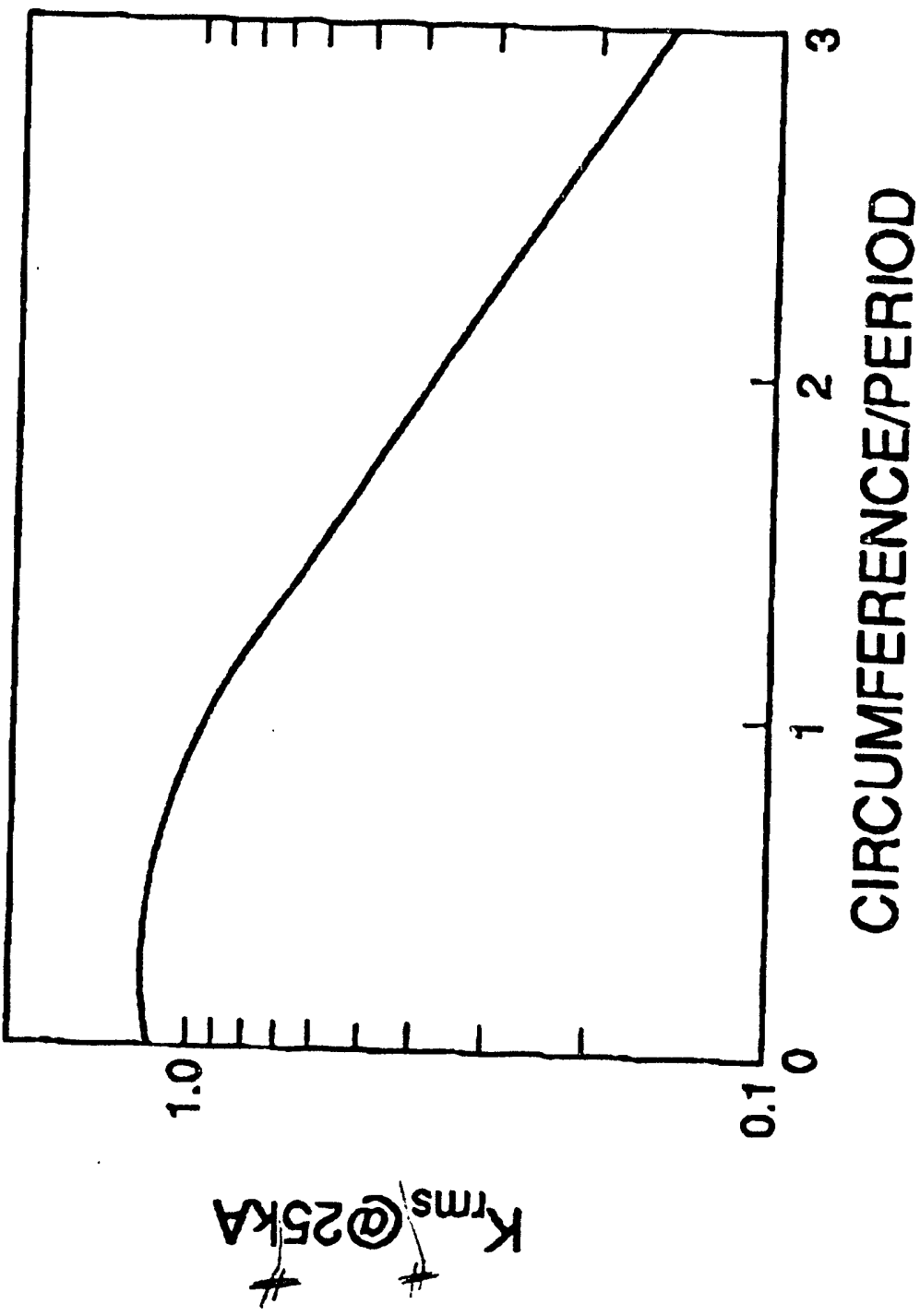




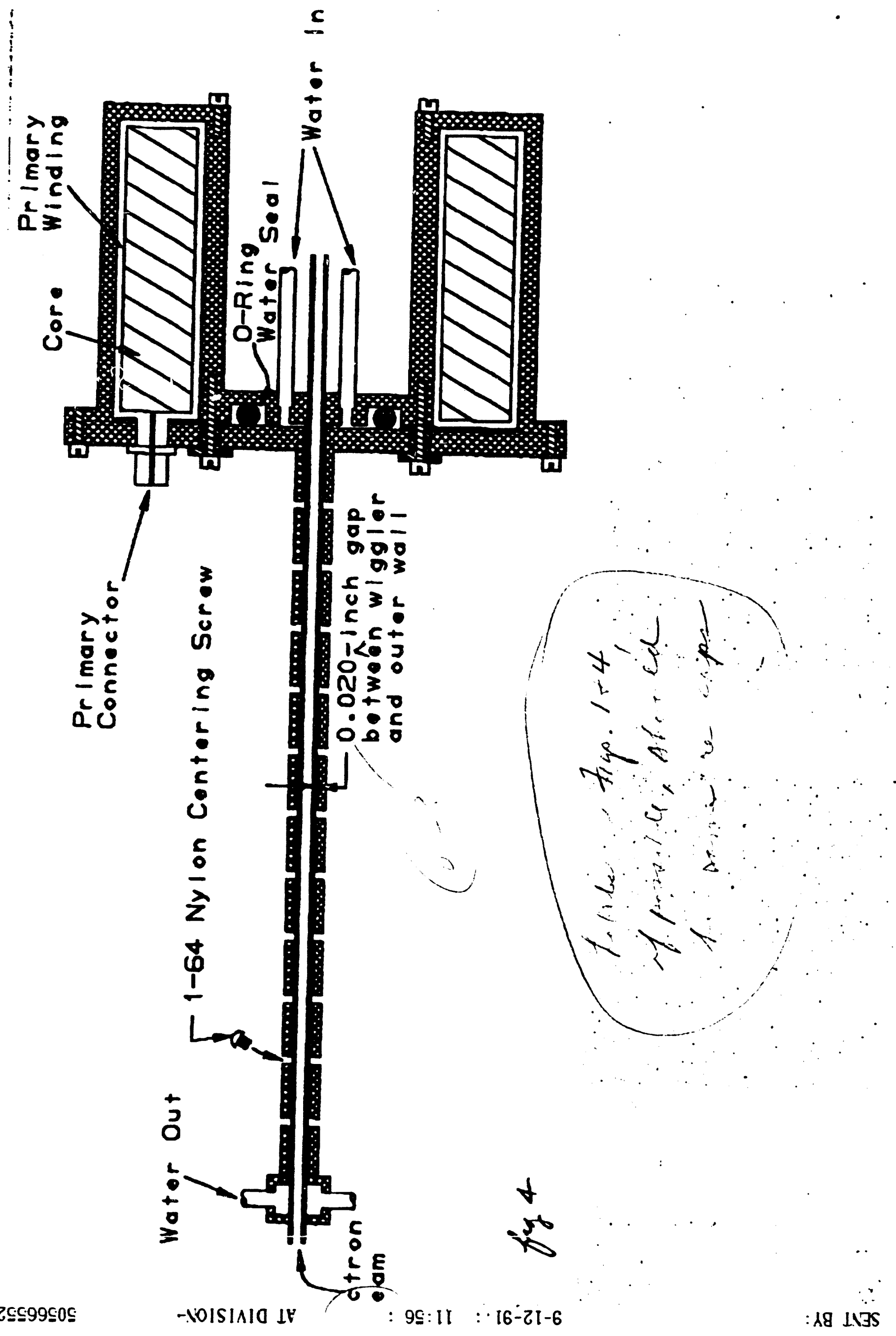




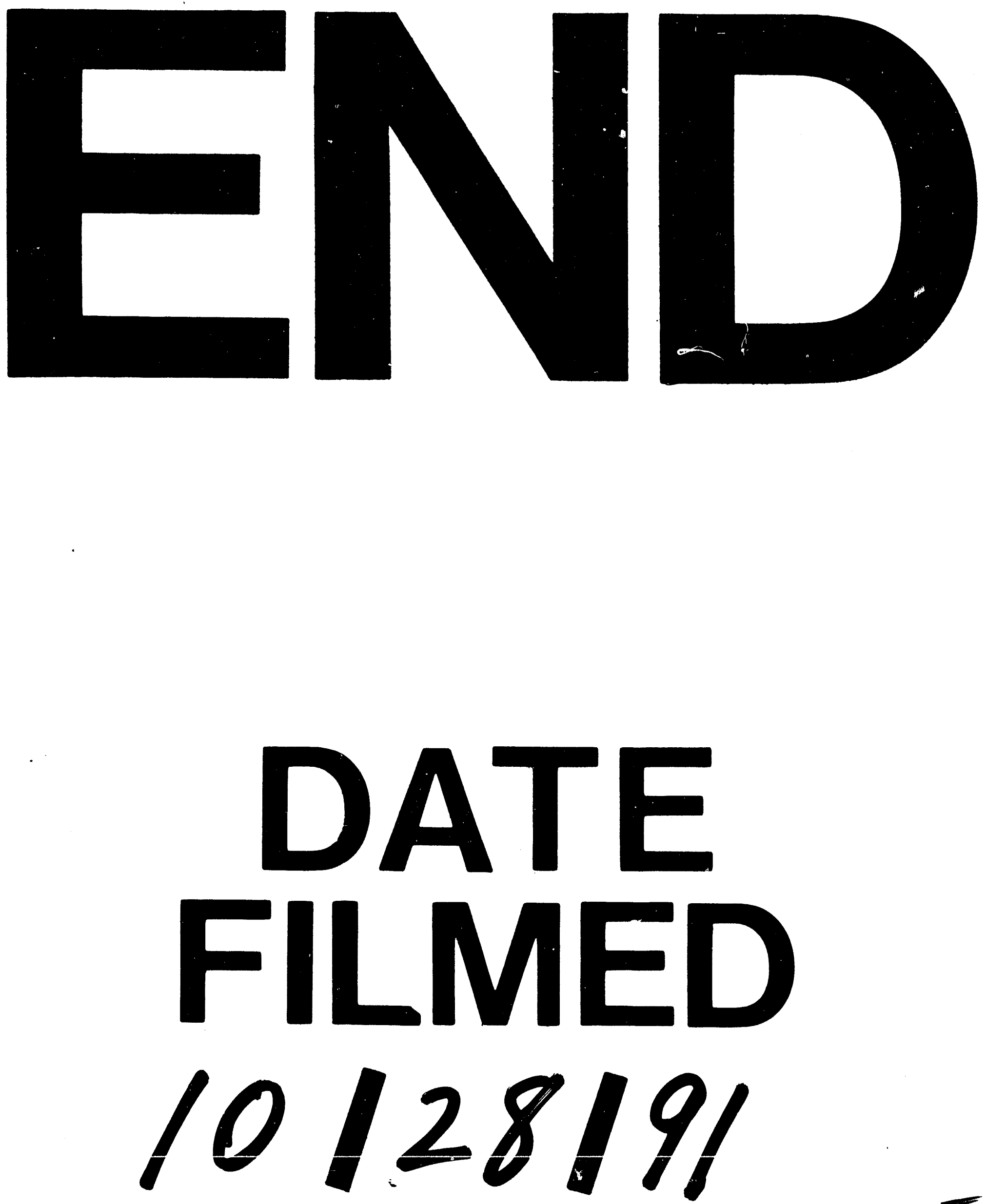
